\title{
Group Recommender Systems Based on Members' Preference for Trusted Social Networks
}

\author{
Xiangshi Wang, Lei Su (D), Qihang Zhou, and Liping Wu \\ School of Information and Automation, Kunming University of Science and Technology, Kunming, China \\ Correspondence should be addressed to Lei Su; s28341@hotmail.com
}

Received 3 December 2019; Revised 15 January 2020; Accepted 1 May 2020; Published 19 May 2020

Academic Editor: Yin Zhang

Copyright (c) 2020 Xiangshi Wang et al. This is an open access article distributed under the Creative Commons Attribution License, which permits unrestricted use, distribution, and reproduction in any medium, provided the original work is properly cited.

\begin{abstract}
With the development of the Internet of Things (IoT), the group recommender system has also been extended to the field of IoT. The entities in the IoT are linked through social networks, which constitute massive amounts of data. In group activities such as group purchases and group tours, user groups often exhibit common interests and hobbies, and it is necessary to make recommendations for certain user groups. This idea constitutes the group recommender system. However, group members' preferences are not fully considered in group recommendations, and how to use trusted social networks based on their preferences remains unclear. The focus of this paper is group recommendation based on an average strategy, where group members have preferential differences and use trusted social networks to correct for their preferences. Thus, the accuracy of the group recommender system in the IoT and big data environment is improved.
\end{abstract}

\section{Introduction}

With the advent of the $5 \mathrm{G}$ era [1], which promotes the development of mobile Internet and big data, users are faced with massive amounts of data on the Internet. It is difficult for search engines to accurately obtain information resources that meet their own needs and personalized preferences. Information overload issues are increasingly prominent [2]. The recommender system [3] is considered to be effective in dealing with this problem. In recent years, research on recommender systems has developed very rapidly. There are several types of recommender systems, such as mobile recommender systems, context-aware recommender systems, and social network recommendation systems. However, these recommender systems can only be recommended for a single user. In real life, there are many situations where we interact mostly with groups, such as while watching a movie, having dinner, and planning a vacation with friends. As such, the recommender system must consider the preferences of each user in the group. This recommender system is called a group recommender system [4]. However, most of these systems deal with every individual preference in the same way, ignoring the personality of each member and the relationships among group members. People have linked entities in the IoT, which has developed rapidly through social networks. Personal social networks, where users imple-ment network interactions, are mainly divided into strong relationship networks and weak relationship networks. A strong relationship network mainly contains applications such as QQ and WeChat. Weak relationship networks include Weibo and various forums. Network members with strong connections have a high degree of relationship with each other, and they are more willing to share their views and experiences without reservation, while weak connections are the opposite. A previous study found that the higher the degree of the relationship between users is, the more the trust exists between users [5]. According to Mui, trust is the subjective expectation of one subject for its future behavior decisions based on its historical interaction experience with another subject [6].

The social network relationship of group members is an essential factor in a group recommender system. Studies have shown that users are more willing to accept 
recommendations from trusted users than recommendations from anonymous users [7]. For example, users are more likely to accept recommendations from friends rather than from strangers. Group-recommended social network factors mainly include personality and trust [8,9]. The higher users' personality leads to less effect by others. Users' trust in another user influences their preferences. The group recommendation studies bases on social networks where users may need to change their preferences to reach group consensus. In social network-based group recommendations, users may need to change their preference to reach a group consensus. At this point, the trust between the members of the group becomes the main influencing factor. However, changing your preference can easily lead to preference differences within the group. Chen e al. [10] uses the similarity of preferences and the relevance of trust, calculating the trust and influence of group members according to the similarity of group members. And finally it gets the final rating through the weighted mean fusion strategy. At the same time, not all group members have significant social networking relationships. Furthermore, according to the users' social network, closely connected users naturally come together to form a user group, and the connections between different user groups are sparse, thus forming a community structure [11]. So this community structure formed by natural social network relationship is a natural grouping method with good interpretability. However, this kind of social network relationship can only achieve a better group recommendation effect when the user relationship information is dense. In the case of sparse user data in the big data environment, the group recommendation is easy to generate a cold start and recommend the group. The improvement associated with this effect is not obvious. It is a challenge in group recommendation problem to address member preferences, random groupings, user relationship sparseness, and cold starts in a social network group recommendation in a big data environment. This paper proposes members' preference for trusted social networks. The system analyzes the group recommendations of the average strategy. When the group recommendations of the average strategy differ greatly in preferences, the trusted social network of the group members is introduced to modify the preferences in the group, to obtain better recommendation results.

The structure of this paper is as follows. Section 2 introduces related work on the social network recommendation and group recommendation methods, and Section 3 elaborates the method based on a preference for trusted social networks proposed in this paper. Section 4 introduces the experimental results. Section 5 summarizes the full text and discusses future work.

\section{Related Work}

2.1. Group-Recommended User Preferences. The recommended method for groups is usually to obtain the preferences of each user in the group [4]. It is generally believed that preferences are used to describe the ordering relationship of decision-makers to two or more items [12]. In the group recommendation, the user's preference acquisition methods are mainly divided into explicit preference acquisitions [13] and implicit preference acquisitions [14]. In the display of preference acquisitions, the user is required to explicitly provide preference information, which is usually defined by a rating on a given interval. MusicFX [15] requires users to rate different music style gen-res and group recommendations based on user ratings. Literature [16] requires users to rate the location, amount of food, and taste of the restaurant. In the implicit preference acquisition method, the user does not need to explicitly provide preference information, but users use historical behavior data to mine user preferences. Crossen et al. [13] learn users' musical preferences from users' music listening behavior data. Let's Browse [17] proposes to use the term frequencyinverse document frequency (TF-IDF) algorithm to learn users' preferences for news topics based on the keywords on users' homepages. As long as there is enough user behavior data, the implicit preference acquisition method can accurately extract users' preference characteristics, which is beneficial to protect the users' privacy.

\subsection{Group Recommendation Preference Fusion Method.} Preference fusion occurs at different stages of the group recommendation process, such that the content of the fusion is different. Literature [18] analyzed 10 preference fusion strategies in detail. The different manifestations of the four most commonly used average strategies are listed below. Assuming that each member's weight is the same, the average of the scores of all group members is used as the recommended score for the group.

Assume that each member's weight is the same in the average strategy group recommendation based on item similarity. The ratings of all group members are averaged as the recommended rating for the group:

$$
\operatorname{ISpre}(G, i)=\frac{1}{|G|} \sum_{u \in G} \operatorname{Items} \operatorname{Pro}(u, i),
$$

where $|G|$ indicates the size of the group; $\operatorname{Items} \operatorname{Pro}(u, i)$ indicates the rating of item $i$ by user $u$ in the recommendation method based on the user similarity; and ISpre $(G, i)$ represents the final forecast score formed by the group recommendations based on the item similarity average strategy.

It is assumed that each member has the same weight in the average strategy group recommendation based on matrix factorization. The average of the ratings of all group members is used as the recommended rating for the group:

$$
\operatorname{MFpre}(G, i)=\frac{1}{|G|} \sum_{u \in G} \operatorname{MatrixfPro}(u, i) \text {, }
$$

where $|G|$ indicates the size of the group; MatrixfPro $(u, i)$ represents the rating of item $i$ by user $u$ in the recommendation method based on matrix factorization; and $\operatorname{MFpre}(G, i)$ represents the final prediction rating formed by the group recommendation based on the matrix factorization average strategy. 
In the average strategy group recommendation based on the popularity of the item, it is assumed that each member has the same weight and the average of the ratings of all group members is used as the recommended rating of the group:

$$
\operatorname{IPpre}(G, i)=\frac{1}{|G|} \sum_{u \in G} \operatorname{ItempPro}(u, i),
$$

where $|G|$ indicates the size of the group; $\operatorname{ItempPro}(u, i)$ represents the rating of item $i$ by user $u$ in the recommendation method based on the popularity of the item; IPpre $(G, i)$ represents the final predicted rating formed by the group recommendation based on the popularity of the item average strategy.

In the average strategy group recommendation based on implicit feedback, it is assumed that each member has the same weight. In addition, the average of the ratings of all group members is used as the group recommendation rating:

$$
\operatorname{IFpre}(G, i)=\frac{1}{|G|} \sum_{u \in G} \operatorname{ImplicitfPro}(u, i),
$$

where $|G|$ indicates the size of the group; $\operatorname{ImplicitfPro}(u, i)$ represents the rating of item $i$ by user $u$ in the recommendation method based on the implicit feedback dataset; and IFpre $(G, i)$ represents the final predicted rating based on group recommendations for implicit feedback dataset average strategy.

\subsection{Degree of Preference Divergence in the Recommendation of} the Average Strategy Group Recommendation. Recommendations generated in the group recommendation of the average strategy [19] may cause dissatisfaction among individual group members, which is known as pain problems. To measure this "pain," the preference divergence is introduced in this paper. Because the chosen group recommendation algorithms are different, the degree of divergence in the group recommendation is different:

$$
\operatorname{ISdis}(G, i)=\frac{1}{|G|}[\operatorname{ISpro}(u, i)-\operatorname{ISmean}(G, i)]^{2},
$$

where $\operatorname{ISpro}(u, i)$ indicates the rating of each user in the group calculated by the recommendation method based on the item similarity; $\operatorname{ISmean}(G, i)$ represents the average of the user ratings in the group; and ISdis $(G, i)$ represents the extent to which members of the group disagree with item $i$ in group $G$.

$$
\operatorname{MFdis}(G, i)=\frac{1}{|G|}[\operatorname{MFpro}(u, i)-\operatorname{MFmean}(G, i)]^{2} .
$$

where $\operatorname{MFpro}(u, i)$ represents the rating for each user in the group calculated by the recommendation method based matrix factorization; MFmean $(G, i)$ represents the average of the user ratings in the group; and $\operatorname{MFdis}(G, i)$ represents the extent to which members of the group disagree with item $i$ in group $G$ :

$$
\operatorname{IPdis}(G, i)=\frac{1}{|G|}[\operatorname{IPpro}(u, i)-\operatorname{IPmean}(G, i)]^{2},
$$

where IPpro $(u, i)$ represents the rating of each user on the item, which can be calculated by the recommendation method of the item popularity; $\operatorname{IPmean}(G, i)$ represents the average of user ratings in the group; and $\operatorname{IPdis}(G, i)$ represents the extent to which members of the group disagree with item $i$ in group $G$ :

$$
\operatorname{IFdis}(G, i)=\frac{1}{|G|}[\operatorname{IFbPro}(u, i)-\operatorname{IFmean}(G, i)]^{2},
$$

where $\operatorname{IFbPro}(u, i)$ represents the rating of each user on the item, which can be calculated by the recommendation method of the implicit feedback dataset; IFmean $(G, i)$ represents the average of the user ratings in the group; and IFdis $(G, i)$ represents the extent to which members of the group disagree with item $i$ in group $G$.

\section{Group Recommender System-Based Members' Preference for Trusted Social Networks}

In group recommender system-based members' preference for trusted social networks, in order to alleviate the problems of data sparsity and cold starts, first, the recommendation methods based on item similarity [3], matrix factorization [20, 21], item popularity, and implicit feedback datasets [22] are adopted to form personalized recommendations for users. The purpose of personalized recommendations is to alleviate data sparsity and cold start problems in group recommendations. Through personalized recommendation, the preference scoring data of trusted members are supplemented. Then, a group recommendation based on the average strategy is adopted for the above recommendation methods. Average strategy is the most commonly used preference fusion strategy in a group recommendation system, which takes the average score of the group members as the score of the group [4]. However, recommendations generated by the average strategy may cause dissatisfaction among individual group members, namely, the so-called preference divergence problem [4]. To avoid this "preference divergence" problem, the degree of preference divergence is calculated by preference differences. Taking preference divergence as a criterion, trust-based social networks are introduced when the preference divergence of group members is greater than that of the group as a whole. In the trusted social network of group members, each group member has several trust members. When the preferences of group members are quite different, the group members can appropriately modify the ratings of group members through the preference ratings of trust members.

\subsection{Calculation of Correction Factors in Group Preference}

3.1.1. Calculation of Correction Factors According to the Divergence Degree. After the group recommendation system based on the average strategy [19] presented in this 
paper, we take the divergence degree as a measure and obtain $\mu:$

$$
\mu=\frac{1}{|G|} F\left[\sum_{u \in G}\left(\operatorname{somepro}(u, i)-\frac{\sum_{v \in G} \operatorname{somepro}(v, i)}{|G|}\right) \geq \sum_{i=3}^{i=11} \operatorname{somedis}(G, i)\right] \text {, }
$$

where $|G|$ represents the number of members of the group and introduces the function $F(x)$. If the expression of $x$ is true, it is counted as 1 . The expression of $x$ does not hold; it is recorded as 0 . somepro $(u, i)$ indicates the user $u$ on $i$ in the above four personalized recommendation methods. somedis $(G, i)$ indicates the divergence degrees obtained in the group recommendations of different average strategies. $\mu$ is a measure of divergence. The larger the value of $\mu$ is, the greater the difference between the preference in the group and the preference recommended by the group of average strategy, and the more need there is to revise the preference of the group members based on the trusted members of the trusted social network. The smaller the value of $\mu$ is, the smaller the difference between the preference in the group and the preference recommended by the group of Average Strategy. When $\mu$ is sufficiently small, it shows that there is no preference divergence in the group recommendation based on the average strategy. As such, it follows the group recommendation based on the average strategy.

3.1.2. Calculation of Correction Factors According to the Standard Value. Through the above analysis of the divergence degree, the divergence degree between the group members and the group recommendation of the average strategy is reduced, and the group recommendation is further optimized. To further study the correction factor $\mu$, finding the rating of the group members for a certain item is proposed. In this rating, we use the sample standard deviation of the scored item, the median of the evaluation range, and the median of the scored item. We, respectively, use standard ${ }_{\text {mean }}$, standard $\mathrm{m}_{\text {middle }}$, and standard median $_{\text {to }}$ to express these three standard values:

$$
\mu_{\text {mean }}=\frac{\left(\text { higher }_{\text {sdui }}-\text { fewer }_{\text {sdui }}\right)}{w \text { standard }_{\text {mean }}}
$$

where standard $\mathrm{d}_{\text {mean }}$ means that the standard deviation of the sample is taken as the standard value. The standard deviation of the sample is calculated through the calculation of the rating. In the item being scored, the score must have a portion larger than the sample standard and a portion smaller than the sample standard deviation. The sample variance is taken as a measure. There are a large number of parts and a small number of parts that are greater than or less than the standard deviation of the sample. The large number of parts is written as higher ${ }_{\text {sdui, }}$, and the small number is written as fewer ${ }_{\text {sdui }}$. In this experiment, in order to better combine the recommendation system of the average strategy with trusted social networks, the parameter $w$ is taken as 2:

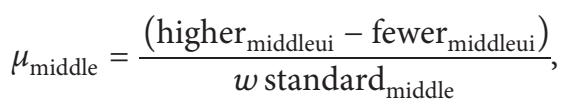

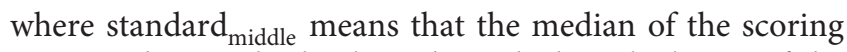
range is the standard value. Through the calculation of the scoring range, the median of the scoring range is calculated. higher $_{\text {middleui }}$ indicates a set larger than the median of the

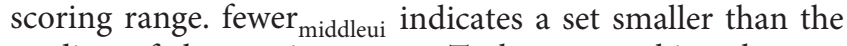
median of the scoring range. To better combine the recommendation system of average strategy with the trusted members in trusted social networks, $w$ is herein taken as 2:

$$
\mu_{\text {median }}=\frac{\left(\text { higher }_{\text {medianui }}-\text { fewer }_{\text {medianui }}\right)}{w f\left(w \text { standard }_{\text {median }}\right)},
$$

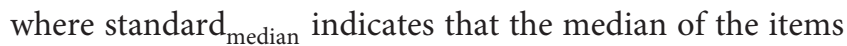
to be scored is taken as the standard value. Through the calculation of the median score, the median of the items to be scored is calculated. higher ${ }_{\text {medianui }}$ indicates the number of sets greater than the median of the item being scored. fewer $_{\text {medianui }}$ represents the number of sets that are less than the median of the item being scored. To better combine the recommendation system of average strategy with the preferences of trusted members in trusted social networks, $w$ is herein taken as 2 .

3.1.3. Calculation of Preference Rating for Trusted Network Members. By trusting the social network, the group members are connected with the members who trust the social network. Each user has one or more trust objects. There are two points to consider for trusting social networks: (a) the trust degree and real preference evaluation of members in trust social networks; and (b) the number of trusted social network members. The formulae are as follows:

$$
\begin{aligned}
\operatorname{ISTR}(\mathrm{TR}, i) & =\frac{\sum_{v \in R} X_{u, v} \cdot \operatorname{ISrating}(v, i)}{\sum_{v \in R} G_{\text {Istrust }}}, \\
\operatorname{MFTR}(\mathrm{TR}, i) & =\frac{\sum_{v \in R} X_{u, v} \cdot \operatorname{MFrating}(v, i)}{\sum_{v \in R} G_{\text {Mftrust }}}, \\
\operatorname{IPTR}(\mathrm{TR}, i) & =\frac{\sum_{v \in R} X_{u, v} \cdot \operatorname{IPrating}(v, i)}{\sum_{v \in R} G_{\text {Iptrust }}}, \\
\operatorname{IFTR}(\mathrm{TR}, i) & =\frac{\sum_{v \in R} X_{u, v} \cdot \operatorname{IFrating}(v, i)}{\sum_{v \in R} G_{\text {IFtrust }}},
\end{aligned}
$$

where $X_{u, v}$ indicates the trust degree of user $u$ to $v . X_{u, v} \in[0$, 1], 0 means distrust and 1 means trust; ISrating $(v, i)$ indicates the true rating of item $i$ by user $v$ in the 
recommendation method based on item similarity in the trust network; MFrating $(v, i)$ indicates the true rating of item $i$ by user $v$ in the matrix factorization based recommendation method in the trust network; IPrating $(v, i)$ indicates the real rating of item $i$ by user $v$ in the recommendation method based on the popularity of the item in the trusted social network; IFrating $(v, i)$ indicates the real rating of item $i$ by user $v$ in the recommendation method based on implicit feedback dataset in the trusted social network; $G_{\text {Istrust }}$ indicates a single trusted user of a group member in a recommendation method based on item similarity; $G_{\mathrm{Mftrust}}$ denotes a single trusted user of a group member in a matrix factorization based recommendation method; $G_{\text {Iptrust }}$ indicates a single trusted user of a group member in a recommendation method based on the popularity of an item; and $G_{\text {IFtrust }}$ represents a single trusted user of a group member in a recommendation method based on an implicit feedback dataset. These parameters are introduced into the group recommendations based on group recommender systems based on members' preference for trusted in social networks. Even in group recommendation without the average strategy, data sparsity and cold start problems in group recommendations can be alleviated to some extent by trusting $\operatorname{ISTR}(\mathrm{TR}, i)$, MFTR (TR, $i$ ), IPTR (TR, $i$ ), and IFTR (TR, $i$ ) in social network.

3.2. Group Recommendation Method Based on Members' Preference for Trusted Social Network Item Similarity. In the group recommendation based on the preference of members who trust social network item similarity, first, the recommendation method based on item similarity is used to generate personalized recommendations for users, and groups are randomly divided to make group recommendations based on the average strategy of item similarity. Through the group recommendation, the following methods are proposed to solve the problem of preference divergence in group recommendation of the average strategy:

$$
\operatorname{ISpre}(G, i)=\left\{\begin{array}{l}
\left|\frac{1}{2}-\mu_{i}^{2}\right| \cdot \operatorname{ISGP}(G, i)+\mu_{i} \cdot \operatorname{ISTR}(\mathrm{TR}, i) \\
\operatorname{ISGP}(G, i), \quad \text { if } \operatorname{ISTR}(\operatorname{TR}, i)=\varnothing, \\
\operatorname{ISTR}(\mathrm{TR}, i), \quad \text { if } \operatorname{ISGP}(G, i)=\varnothing,
\end{array}\right.
$$

where ISGP $(G, i)$ (item similar group prediction) indicates a group recommendation based on item similarity average strategy; ISTR $(\mathrm{TR}, i)$ (item similarity trust rating) indicates the rating of recommendation methods that trust the similarity of items of social network members; and $\mu_{i}$ indicates that the divergence degree or standard value is obtained as correction factors. The function of the correction factor is to reduce the problem of preference divergence among group members in the average strategy. ISpre $(G, i)$ (item similarity prediction) indicates a group recommendation based on the preference of members who trust the similarity of social network items. If $\operatorname{ISTR}(\mathrm{TR}, i)=\varnothing$, the members of the group do not trust the network. At this time, we adopt the group recommendation based on the average strategy of item similarity. If $\operatorname{ISGP}(G, i)=\varnothing$, there is no exact group recommendation for the group recommendation of average strategy; then, a recommendation based on the trusted social network is adopted.

\subsection{Group Recommendation Method Based on Members'} Preference for Trusted Social Network Matrix Factorization. In the group recommendation based on the trust social network matrix factorization preference, the recommendation method based on matrix decomposition is used to generate personalized recommendations for the user. The group is randomly divided, and the group is recommended based on the matrix factorization average strategy. After the group recommendation, for the divergence problem in the group recommendation of the average strategy, the following methods are proposed:

$$
\operatorname{MFpre}(G, i)=\left\{\begin{array}{l}
\left|\frac{1}{2}-\mu_{i}^{2}\right| \cdot \operatorname{MFGP}(G, i)+\mu_{i} \cdot \operatorname{MFTR}(\mathrm{TR}, i) \\
\operatorname{MFGP}(G, i), \quad \text { if } \operatorname{MFTR}(\mathrm{TR}, i)=\varnothing, \\
\operatorname{MFTR}(\mathrm{TR}, i), \quad \operatorname{if} \operatorname{MFGP}(G, i)=\varnothing,
\end{array}\right.
$$

where MFGP $(G, i)$ (matrix factorization group prediction) indicates a group recommendation based on the matrix factorization average strategy; MFTR $(\mathrm{TR}, i)$ (matrix factorization trust rating) indicates the score of the recommendation method based on matrix factorization of trusted social network member preferences; and $\mu_{i}$ indicates that the divergence degree or the standard value is obtained as a correction factor. The function of the correction factor is to correct the preference differences among the group members in the group recommendation of the average strategy. MFpre $(G, i)$ (matrix factorization prediction) indicates a group recommendation based on trusted social network matrix factorization member preferences. If $\operatorname{MFTR}(\mathrm{TR}, i)=\varnothing$, the group members do not trust the social network. In this case, the average strategy group recommendation based on matrix factorization is adopted. If $\operatorname{MFGP}(G, i)=\varnothing$, no group recommendation is formed in the random group. At this time, the recommendation based on the trusted social network is adopted.

\subsection{Group Recommendation Method Based on Members'} Preference for Trusted Social Network Item Popularity. In group recommendations based on trusted social network item popularity membership preferences, personalized recommendations are generated for users by the recommendation method based on the popularity of the item. Then, the groups are randomly divided and group recommendations are made based on the average strategy of the popularity of the item. After the group recommendation, the following methods are proposed to solve the problem of preference divergence in the group recommendation based 
on the average strategy of trusted social network item popularity:

$$
\operatorname{IPpre}(G, i)=\left\{\begin{array}{l}
\left|\frac{1}{2}-\mu_{i}^{2}\right| \cdot \operatorname{IPGP}(G, i)+\mu_{i} \cdot \operatorname{IPTR}(\mathrm{TR}, i) \\
\operatorname{IPGP}(G, i), \quad \text { if } \operatorname{IPTR}(\mathrm{TR}, i)=\varnothing, \\
\operatorname{IPTR}(\mathrm{TR}, i), \quad \text { if } \operatorname{IPGP}(G, i)=\varnothing .
\end{array}\right.
$$

where IPGP $(G, i)$ (item popular group prediction) indicates the average strategy group recommendation based on the popularity of the item; IPTR $(\mathrm{TR}, i)$ (item popular trust rating) indicates a recommended method for trusting the popularity of the social networking item; $\mu_{i}$ is the divergence degree or standard value obtained and used as a correction factor to correct the preference divergence problem among group members in group recommendation based on the average strategy of item popularity; and IPpre $(G, i)$ (item popular prediction) indicates a group recommendation based on member preference of trusted social network item popularity. If $\operatorname{IPTR}(\mathrm{TR}, i)=\varnothing$, the members of the group do not trust the social network, and the average strategy group recommendation based on the popularity of the item is adopted. This means that no group recommendation based on the average strategy of the popularity of the item has been formed; thus, the recommendation based on the trusted social network will be adopted.

\subsection{Group Recommendation Method Based on Implicit} Feedback of the Dataset of Members' Preference by Trusted Social Networks. In group recommendations based on implicit feedback of member preferences by trusted social networks, a recommendation method based on implicit feedback dataset is used to generate personalized recommendations for users, groups are randomly divided, and group recommendations based on the average strategy of implicit feedback datasets are carried out on the groups. After group recommendation, the following methods are proposed to solve the preference divergence problem in the group recommendation based on the implicit feedback dataset average strategy:

$$
\operatorname{IFpre}(G, i)=\left\{\begin{array}{l}
\left|\frac{1}{2}-\mu_{i}^{2}\right| \cdot \operatorname{IFGP}(G, i)+\mu_{i} \cdot \operatorname{IFTR}(\mathrm{TR}, i) \\
\operatorname{IFGP}(G, i), \quad \text { if } \operatorname{IFTR}(\mathrm{TR}, i)=\varnothing, \\
\operatorname{IFTR}(\mathrm{TR}, i), \quad \operatorname{if} \operatorname{IFGP}(G, i)=\varnothing,
\end{array}\right.
$$

where IFGP $(G, i)$ (implicit feedback group prediction) indicates the group recommendation of average strategy based on the implicit feedback dataset; IFTR (TR, i) (implicit feedback trust rating) represents recommendations based on implicit feedback datasets of trusted social network members; $\mu_{i}$ is used to find the divergence degree and standard value as correction factors to correct the preference divergence problem in group recommendation based on the implicit feedback dataset average strategy; and IFpre $(G, i)$ (implicit feedback prediction) indicates a group recommendation based on the implicit feedback of dataset member preferences by trusted social networks. If $\operatorname{IFTR}(\mathrm{TR}, i)=\varnothing$, the members of the group do not trust the social network, and the group recommendation based on the implicit feedback dataset average strategy is adopted. If $\operatorname{IFGP}(G, i)=\varnothing$, the average strategy group recommendation based on the implicit feedback dataset has not formed an effective group recommendation; at this time, the recommendation based on the implicit feedback dataset and the trusted social network is adopted.

3.6. Algorithm Based on Members' Preference for Trusted Social Networks. The evaluation dataset is divided into a training set and a test set. After preprocessing the data, all trust-based social networks will be used. At the same time, the following group member preference algorithm is proposed to reduce the preference divergence problem in the group recommendation of average strategy. The basic idea behind the experiment is as follows: using recommendation methods based on item similarity, matrix factorization, item popularity, and implicit feedback dataset, personalized recommendations are generated for users. Groups are divided randomly and $\mu$ is calculated according to the divergence and standard value. Through trusting social networks, trust members are found for team members, after which group recommendations are generated and the MAE is calculated by correcting for the preference differences of trusted social networks. Through trusted social networks, we can find trusted members for team members and can generate group recommendations through correcting the differences in the preferences of trusted social networks. Finally, we calculate MAE. The algorithm is named GRIMPFTSN (group recommendation involves members preference for trusted social networks) Algorithm 1 was proposed and verified by experiments.

\section{Experiment and Analysis}

4.1. Experimental Dataset. The dataset uses the FilmTrust dataset (https://www.librec.net/datasets/filmtrust.zip) [23]. The dataset includes 1508 users, 2071 movies, and 35497 reviews. The density of data is $1.14 \%$. The dataset is preprocessed as follows: users are randomly divided into training sets and test sets according to a certain proportion, $80 \%$ for training sets and $20 \%$ for test sets. Assuming that a user has 10 scores on items, we select 8 of the users as training sets and 2 as test sets. To divide the data, we select users who score items more than 5 times. Through data preprocessing, it can be obtained that there are 1478 training set users and 1420 testing set users. Further, 607 users in the trusted social network have trusted objects through the trusted social network, and $41 \%$ of users in the training set will use trusted social networks. In this paper, a randomized group approach is used for experimental research. The goal is to alleviate the computational cost of the experiment and 


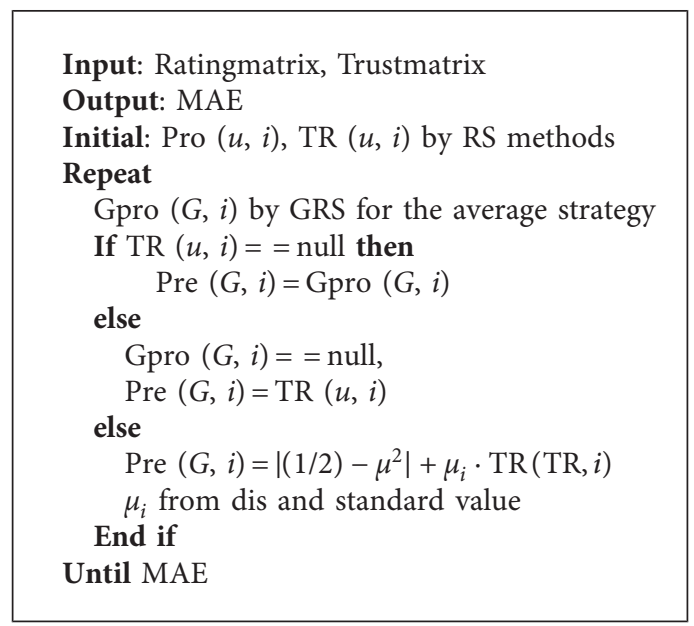

Algorithm 1: GRIMPFTSN.

to verify the validity of the group preference group recommendations of the social networks that trust the randomly generated team.

4.2. Evaluation Method MAE. To evaluate the effectiveness of the group recommendation based on trusted social network preferences, the offline data evaluation method, mean absolute error (MAE) [24], is used to measure the prediction accuracy:

$$
\operatorname{MAE}=\frac{\sum_{i=1}^{N}(\operatorname{pre}(G, i)-\operatorname{real}(G, i))}{N},
$$

where pre $(G, i)$ represents a group recommendation based on trusted social network preferences; real $(G, i)$ represents the real recommendation in the group; and $N$ represents the number of items in the group recommendation.

\subsection{Group Recommendation Experiment Based on Members'} Preference for Trusted Social Network Item Similarity. Experimental description: in Table 1, IS_average represents the group recommendation based on item similarity average strategy (GRBOISAS) and $\mu_{\text {ISdis }}$ indicates the method calculated according to the degree of divergence, named IStrust_dis. Other methods are not applicable to group recommendation based on members' preference for trusted social network item similarity.

In this experiment, we randomly selected 100 times according to the number of the group. The group size is 3-11 people. Each group sampling is relatively independent. We obtained MAE through the experiment. The MAE shows the difference between group recommendation based on member preference for trusted social network item popularity and group recommendations based on the average strategy. As shown in this experiment, we made 100 random selections according to the number in the group. Each group sampling is relatively independent, including 3-11 people. We obtained the MAE through the experiment. MAE is the mean absolute error. The mean absolute error shows the difference between group recommendations based on the
TABLE 1: IS experimental description.

\begin{tabular}{lc}
\hline Name & Description \\
\hline IS_average & GRBOISAS \\
IStrust_dis & $\mu_{\text {ISdis }}$ \\
\hline
\end{tabular}

preference of similar members of trusted social network items and group recommendations based on the average strategy of the trusted social network. The results are shown in Figure 1.

It is apparent in Figure 1 that under the average strategy based on item similarity, the MAE of IS_average decreases as the group increases in size. In the group recommendation based on the item similarity average strategy, there is no accumulation of the user's historical behavior, and it is not a personalized recommendation. Thus, the differences in the recommendation results of all users are very small. When the difference in the users' recommendation results is small, the difference in the group recommendation based on the average strategy formed by the user is also small. In this experiment, the group recommendation based on the average strategy of item similarity is also verified to have a good recommendation effect by randomly dividing the groups. IStrust_dis corrects the preferences of group members in the group recommendation based on the average strategy of item similarity. By revising the preferences of group members, the effect of group recommendation based on the item similarity average strategy is further improved.

4.4. Group Recommendation Experiment Based on Members' Preference for Trusted Social Network Matrix Factorization. The variables in Table 2 are as follows: MF_average is the group recommendation based on the matrix factorization average strategy (GRBOMFAS). $\mu_{\text {MFdis }}$ denotes a method for calculating the correction factor according to the divergence degree, which is named MFtrust_dis; $\mu_{\mathrm{MFss}}$ means that in the calculation of the standard value, the sample standard variance is used to calculate the correction factor, named MFtrust_sd; $\mu_{\text {MFsmiddle means that the standard variance }}$ 


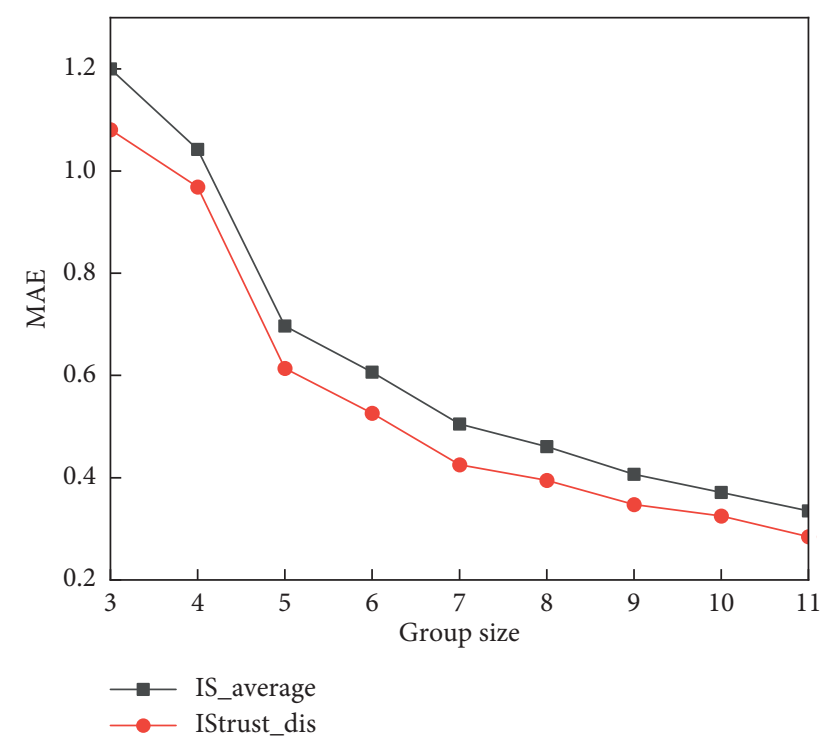

Figure 1: MAE comparison of different group sizes based on the item similarity average strategy.

TABLE 2: MF experimental description.

\begin{tabular}{lc}
\hline Name & Description \\
\hline MF_average & GRBOMFAS \\
MFtrust_dis & $\mu_{\text {MFdis }}$ \\
MFtrust_sd & $\mu_{\text {MFssd }}$ \\
MFtrust_smiddle & $\mu_{\text {MFsmiddle }}$ \\
MFtrust_smedian & $\mu_{\text {MFsmedian }}$ \\
\hline
\end{tabular}

calculation method of samples is adopted in the calculation of the standard value, named MFtrust_smiddle; and $\mu_{\mathrm{MFsmedian}}$ means that in the calculation of the standard value, the calculation method of the median value of the evaluated item is adopted and is named MFtrust_smedian.

In this experiment, we made 100 random selections according to the number of the group, and the group size was 3-11. Each group sampling is relatively independent, and Figure 2 is obtained through experiments. MAE is the mean absolute error. In this experiment, the root mean square error is used to represent the performance difference between the group recommendations based on trusted social network matrix factorization member preferences and group recommendations based on the matrix factorization average strategy.

As shown in Figure 2, when the group size is 3-6, the experimental effect of MFtrust_dis is obviously better than other experimental effects. The performance of the group recommendation based on the average strategy of matrix factorization is greatly improved. When the group size is 7-11, the MAE obtained by the MFtrust_smiddle method is lower, and the group recommendation effect is better.

4.5. Group Recommendation Based on Members' Preference for Trusted Social Network Item Popularity. The variables given in Table 3 are defined as follows: IP_average is a group

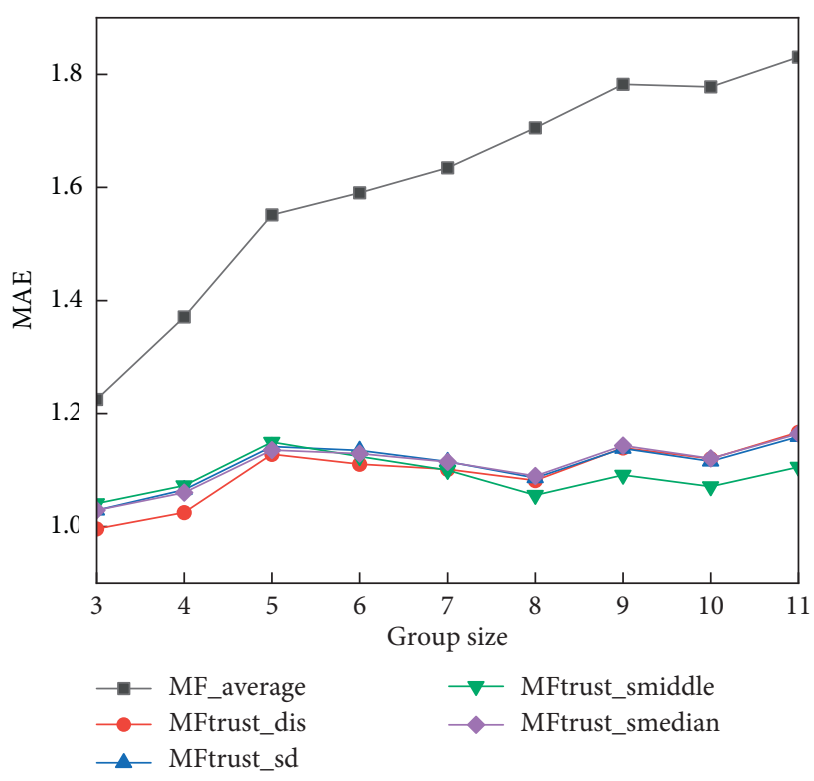

FIgURE 2: MAE comparison of different group sizes based on the matrix factorization average strategy.

recommendation based on item popularity average strategy (GRBOIPAS); $\mu_{\text {IPdis }}$ refers to the method of calculating the correction factor according to the divergence degree, named IPtrust_dis; $\mu_{\text {IPssd }}$ means that in the calculation of the standard value, the sample standard variance is used to calculate the correction factor, named IPtrust_sd; $\mu_{\text {IPsmiddle }}$ means that in the calculation of the standard value, the middle value of the evaluable range is adopted as the calculation method of the standard value, named IPtrust_smiddle; and $\mu_{\text {IPsmedian }}$ indicates that in the calculation of the standard value, the calculation method of the median value of the evaluated article is adopted, named IPtrust_smedian.

In this experiment, we made 100 random selections according to the number of groups, with a group size of $3-11$, for which each group sampling was relatively independent. Figure 3 is obtained through the experiment. In this experiment, MAE represents the effect of group recommendation based on the preference of members of the popularity of the item and the group recommendation based on the average popularity strategy of the item.

As shown in Figure 3, the experimental effect of IPtrust_dis is obviously better than other experimental effects when the group size is 3-4. When the group size is 5-7, the MAE of the IPtrust_smedian method is lower than those of other methods, and the group recommendation effect is better. When the group size is 8-11, the MAE of IPtrust_smiddle is lower than those of other methods, and the group recommendation effect is better.

4.6. Group Recommendation Based on Members' Preference for Trusted Social Network Implicit Feedback Datasets. The variables in Table 4 are defined as follows: IF_average is a group recommendation based on an implicit feedback dataset average strategy (GRBOAIFDAS); $\mu_{\text {IFdis }}$ indicates the method of calculating the correction factor according to the 
TABLE 3: IP experimental description.

\begin{tabular}{lc}
\hline Name & Description \\
\hline IP_average & GRBOIPAS \\
IPtrust_dis & $\mu_{\text {IPdis }}$ \\
IPtrust_sd & $\mu_{\text {IPssd }}$ \\
IPtrust_smiddle & $\mu_{\text {IPsmiddle }}$ \\
IPtrust_smedian & $\mu_{\text {IPsmedian }}$ \\
\hline
\end{tabular}

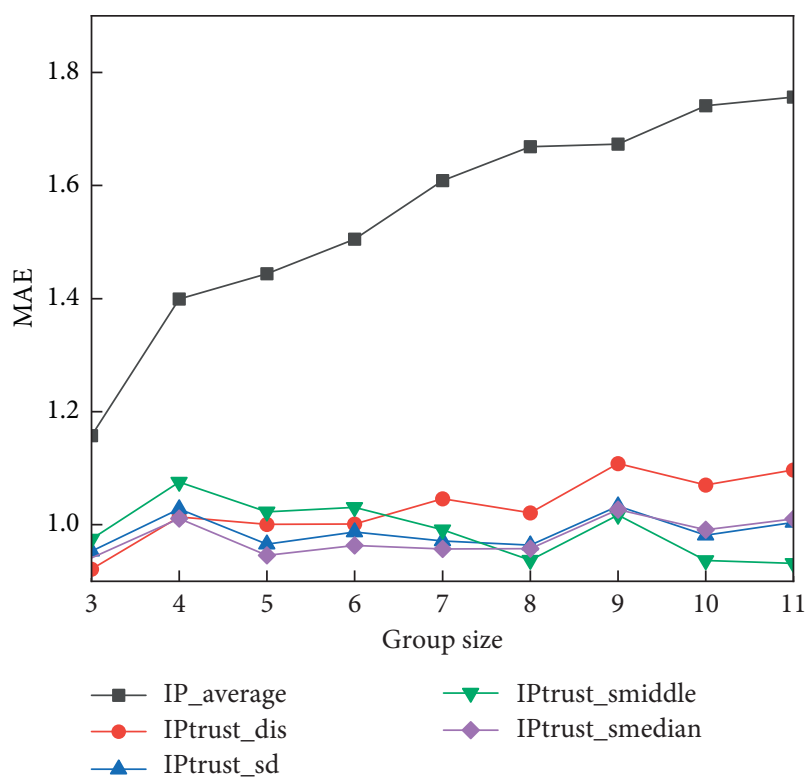

Figure 3: MAE comparison of different group sizes based on the item popularity average strategy.

TABLE 4: IF experimental description.

\begin{tabular}{lc}
\hline Name & Description \\
\hline IF_average & GRBOIPAS \\
IFtrust_dis & $\mu_{\text {IFdis }}$ \\
IFtrust_sd & $\mu_{\text {IFssd }}$ \\
IFtrust_smiddle & $\mu_{\text {IFsmiddle }}$ \\
IFtrust_smedian & $\mu_{\text {IFsmedian }}$ \\
\hline
\end{tabular}

divergence degree, named IFtrust_dis; $\mu_{\text {IFssd }}$ means that in the calculation of the standard value, the sample standard variance is used to calculate the correction factor, named IFtrust_sd; $\mu_{\text {IFmiddle }}$ means that in the calculation of the standard value, the middle value of the evaluable range is used as the calculation method of the standard value, named IFtrust_smiddle; and $\mu_{\mathrm{IF} \text { median }}$ means that in the calculation of the standard value, the method of calculating the median of the evaluated items is adopted, named IFtrust_smedian.

In this experiment, we made 100 random selections according to the number of groups, with a group size of 3-11. Each group sampling was relatively independent. Figure 4 is obtained through the experiment. MAE represents mean absolute error, and MAE represents group recommendation based on the trusted social network implicit feedback dataset member preference and group

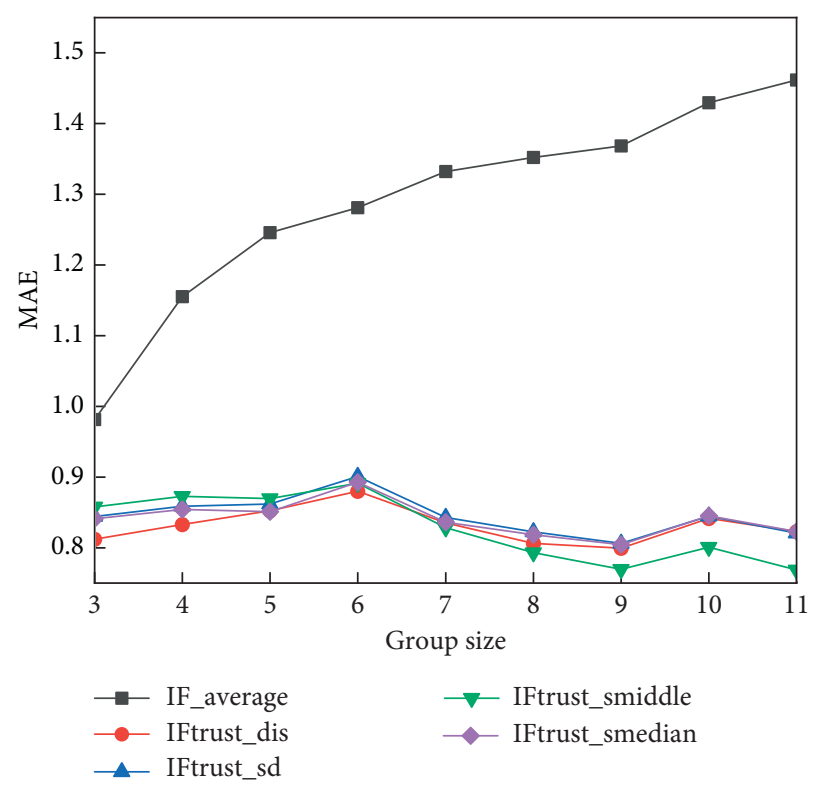

FIGURE 4: MAE comparison of different group sizes based on the implicit feedback dataset average strategy.

TABLe 5: UR experimental description.

\begin{tabular}{lc}
\hline Name & Description \\
\hline IS_rate & SNUBOIS \\
MF_rate & SNUBOMF \\
IP_rate & SNUBOTPOI \\
IF_rate & SNUBOIFD \\
\hline
\end{tabular}

recommendation effect based on the implicit feedback dataset average strategy.

From Figure 4, it is apparent that the experimental effect of IFtrust_dis is obviously better than other experimental effects when the group size is $3-6$. When the group size is 7-11, the MAE of IFtrust_smiddle is lower than that of other methods.

4.7. Utilization Rate of Trusted Social Networks. The variables in Table 5 are defined as follows: IS_rate is the social network utilization based on item similarity (SNUBOIS); MF_rate is the social network utilization based on matrix factorization (SNUBOMF); IP_rate is the social network utilization based on item popularity (SNUBOIP); and IF_rate is the social network utilization based on implicit feedback dataset (SNUBOIFD).

In this experiment, we made 100 random selections according to the number of groups, with a group size of 3-11. Each group sampling was relatively independent. Figure 5 is obtained through the experiment. Percent indicates the utilization rate of social networks, and group size indicates the size of groups.

Figure 5 shows that the utilization rate of trusted social networks is higher when the group is larger, showing a relatively stable trend. Through the above experiments, it is apparent that when the group is bigger, the higher the social network utilization rate. The utilization rate of social 


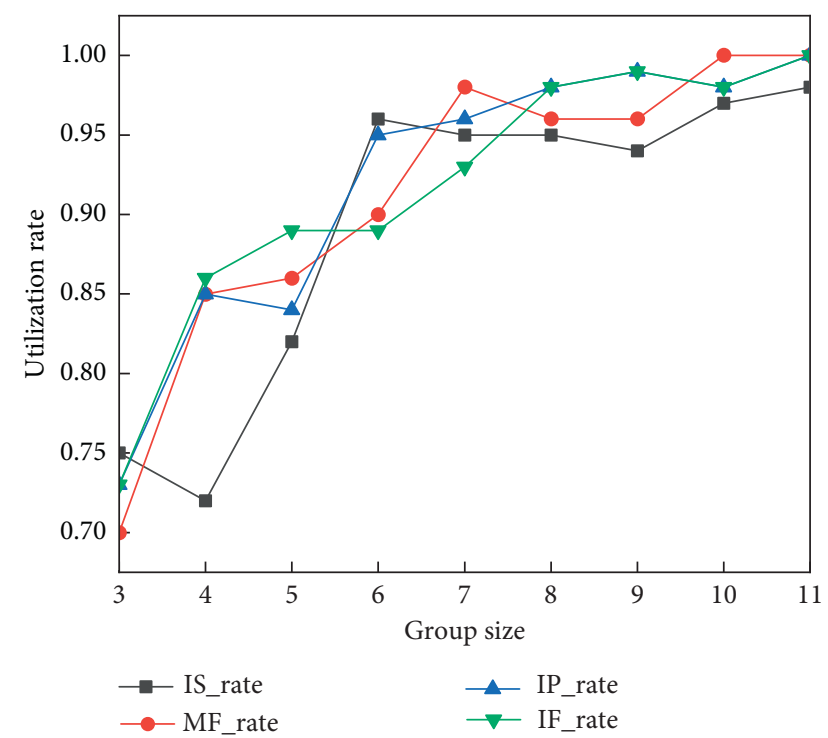

FIGURE 5: Social network utilization rate.

networks shows that the utilization rate of social networks recommended by groups based on the preference of similar members of projects is relatively low. Thus, the average strategy group recommendation approach is more suitable.

\section{Conclusion}

This paper introduces group recommendation based on members' preferences of trusted social networks. Compared with previous experiments, this paper proposes that group recommendation based on trusted social network preferences greatly reduced the MAE and produced an optimal model in the face of different recommendation methods. In this paper, the preferences of group members are modified to reduce the divergence of group members and further optimize the group recommendation of average strategy. By comparing four types of group recommendations based on trusted social networks, it was found that group recommendations based on the preferences of similar members of trusted social networks produced superior results. In this experiment, the item-based recommendation system that has been used in previous experiments is further verified to be suitable for recommendation fusion. Therefore, it is also discovered that the recommendation effect of the members' preference group based on trusted social network project similarity is also optimal. By comparing four types of group recommendation systems based on trusted social network member preferences, it is found that the recommendation system based on trusted social network item popularity has the best interpretability. The effect of this experiment has been greatly improved. And different types of group recommendation systems based on social networks are also compared. However, for each group recommendation system based on trusted social network member preferences, there is no in-depth study, nor does it exactly indicate which exact factors of social network affect the results of group recommendation. It needs to be extended to use in different datasets.

\section{Data Availability}

The FilmTrust dataset used to support the study is available at https://www.librec.net/datasets/filmtrust.zip [23].

\section{Conflicts of Interest}

The authors declare that they have no conflicts of interest.

\section{Acknowledgments}

The work was supported by the National Science Foundation of China under Grant no. 61365010.

\section{References}

[1] K. Zheng, L. Zhao, J. Mei, M. Dohler, W. Xiang, and Y. Peng, "10 Gb/s hetsnets with millimeter-wave communications: access and networking-challenges and protocols," IEEE Communications Magazine, vol. 53, no. 1, pp. 222-231, 2015.

[2] H.-L. Xu, X. Wu, X.-D. Li, and B.-P. Yan, "Comparison study of Internet recommendation system," Journal of Software, vol. 20, no. 2, pp. 350-362, 2009.

[3] P. Resnick and H. R. Varian, "Recommender systems," Communications of the ACM, vol. 40, no. 3, pp. 56-58, 1997.

[4] I. Garcia, S. Pajares, L. Sebastia, and E. Onaindia, "Preference elicitation techniques for group recommender systems," Information Sciences, vol. 189, pp. 155-175, 2012.

[5] W. Wang and I. Benbasat, "Attributions of trust in decision support technologies: a study of recommendation agents for e-commerce," Journal of Management Information Systems, vol. 24, no. 4, pp. 249-273, 2008.

[6] L. Mui, Computational Models of Trust and Reputation: Agents, Evolutionary Games, and Social Networks, Massachusetts Institute of Technology, Cambridge, MA, USA, 2002.

[7] M. O'Connor, D. Cosley, J. A. Konstan, and J. Riedl, "PolyLens: a recommender system for groups of users," in ECSCW, pp. 199-218, Springer, Berlin, Germany, 2001.

[8] H. D. Ambulkar and A. Pathan, "Recommender system challenges and methodologies in social network: survey," International Journal of Science and Research (IJSR), vol. 4, no. 11, pp. 286-289, 2015.

[9] M. Tavakolifard and K. Almeroth, "Social computing: an intersection of recommender systems, trust/reputation systems, and social networks," IEEE Network, vol. 26, no. 4, pp. 53-58, 2012.

[10] Y.-L. Chen, L.-C. Cheng, and C.-N. Chuang, "A group recommendation system with consideration of interactions among group members," Expert Systems with Applications, vol. 34, no. 3, pp. 2082-2090, 2008.

[11] M. Girvan and M. E. J. Newman, "Community structure in social and biological networks," Proceedings of the National Academy of Sciences, vol. 99, no. 12, pp. 7821-7826, 2002.

[12] L.-C. Wang, X.-W. Meng, and Y.-J. Zhang, "Context-aware recommender systems," Journal of Software, vol. 23, no. 1, pp. 1-20, 2012.

[13] A. Crossen, B. Jay, and K. J. Hammond, "Flytrap: intelligent group music recommendation," in Proceedings of the 7 th International Conference on Intelligent User Interfaces, pp. 184-185, San Francisco, CA, USA, January 2002.

[14] Y. Dunham, A. S. Baron, and M. R. Banaji, "The development of implicit intergroup cognition," Trends in Cognitive Sciences, vol. 12, no. 7, pp. 248-253, 2008. 
[15] J. F. McCarthy and T. D. Anagnost, "MUSICFX: an arbiter of group preferences," in Proceedings of the AAAI Spring Symposium on Intelligent Environments, Menlo Park, CA, USA, 1998.

[16] D. Ribeiro Soriano, "Customers' expectations factors in restaurants," International Journal of Quality \& Reliability Management, vol. 19, no. 8-9, pp. 1055-1067, 2002.

[17] H. Lieberman, N. W. Van Dyke, and A. S. Vivacqua, "Let's browse: a collaborative web browsing agent," IUI, vol. 99, pp. 65-68, 1999.

[18] S. Amer-Yahia, S. B. Roy, A. Chawlat, G. Das, and C. Yu, "Group recommendation," Proceedings of the VLDB Endowment, vol. 2, no. 1, pp. 754-765, 2009.

[19] S. Basu Roy, S. Amer-Yahia, A. Chawla, G. Das, and C. Yu, "Space efficiency in group recommendation," The VLDB Journal, vol. 19, no. 6, pp. 877-900, 2010.

[20] Y. Koren, R. Bell, and C. Volinsky, "Matrix factorization techniques for recommender systems," Computer, vol. 42, no. 8, pp. 30-37, 2009.

[21] D. Bokde, S. Girase, and D. Mukhopadhyay, "Matrix factorization model in collaborative filtering algorithms: a survey," Procedia Computer Science, vol. 49, pp. 136-146, 2015.

[22] G. Li and W. Ou, "Pairwise probabilistic matrix factorization for implicit feedback collaborative filtering," Neurocomputing, vol. 204, pp. 17-25, 2016.

[23] G. Guo, J. Zhang, and N. Yorke-Smith, "A novel bayesian similarity measure for recommender systems," in Proceedings of the 23rd International Joint Conference on Artificial Intelligence, IJCAI 2013, pp. 2619-2625, Beijing, China, August 2013.

[24] J. Castro, J. Lu, G. Zhang, Y. Dong, and L. Martinez, "Opinion dynamics-based group recommender systems," IEEE Transactions on Systems, Man, and Cybernetics: Systems, vol. 48, no. 12, pp. 2394-2406, 2018. 